\title{
The effect of insole on the knee valgus angle and pelvic muscle activity during high-intensity weight-bearing tasks
}

\author{
DOI: https://doi.org/10.5114/pq.2021.107848
}

\author{
Seung-Eun Oh${ }^{1}$, Tae-Lim Yoon ${ }^{2}$ \\ ${ }^{1}$ Rehabilitation Center, Cello Hospital, Cheongju, Republic of Korea \\ 2 Department of Physical Therapy, College of Health and Medical Science, Cheongju University, Cheongju, \\ Republic of Korea
}

\begin{abstract}
Introduction. The purpose was to investigate changes in knee valgus angle, as well as associated pelvic muscles, gluteus medius and tensor fasciae latae, when using an insole in people with leg length mismatch less than $2 \mathrm{~cm}$ in high-intensity weight-bearing tasks.

Methods. The knee valgus angle, muscle activity of the gluteus medius, and muscle activity of the tensor fasciae latae were measured with and without an insole during a drop jump task and a step landing task. All measurements were performed 3 times, and the average value was used. The subjects practised 3 times before the measurement and took a sufficient rest between each measurement.

Results. There was no significant difference between the knee valgus angles with or without an insole during the drop jump task or step landing task. For the drop jump task, a significant difference was observed in the muscle activity of the tensor fasciae latae on the short leg side; for the step landing task, a significant difference was noted in the muscle activity of the gluteus medius on the long leg side $(p<0.05)$.

Conclusions. The use of insoles by people with leg length discrepancy during high-intensity landing may prevent non-contact anterior cruciate ligament damage, which is frequently seen in high-intensity landings.
\end{abstract}

Key words: gluteus medius, landing, leg length discrepancy, tensor fasciae latae

\section{Introduction}

Leg length discrepancy is largely classified into 2 types, depending on the cause [1]. The first is actual leg length discrepancy, which is a discrepancy in the structural leg length caused by anatomical or structural changes due to a congenital dysfunction or trauma. The second is functional leg length discrepancy due to altered mechanisms of the lower extremities function, such as contracture of the joints, malalignment of the body, or calcaneal eversion between the feet greater than $3^{\circ}$, which results in compensatory changes [2]. Leg length discrepancy can be caused by congenital or acquired causes [3], and it leads to problems of the musculoskeletal system, such as osteoarthritis of the hip joint, knee pain, back pain, and scoliosis [4], as well as functional problems, such as abnormal walking or loss of balance [5].

Treatment of leg length discrepancy depends on the severity of the discrepancy and of the symptoms [6]. The range of the discrepancy is clinically very important, but there are several opinions as to how big it must be to warrant treatment. Treatment methods include raising the height of the shoes, surgery to lengthen or shorten the leg length, and epiphysiodesis $[5,7]$. When the leg length discrepancy is not large, increasing the height of the shoe on the shorter leg is often used. In general, when the leg length discrepancy is $2 \mathrm{~cm}$ or less, an insole is inserted into the shoe, and when it is $2 \mathrm{~cm}$ or more, a way is found to raise the sole [5]. Surgical treatment may be considered, which is a more reliable method, but it is not generally recommended if the difference in leg length is less than $25 \mathrm{~mm}$ because of the limited advantages provided versus the potential morbidity associated with sur- gery [8]. For smaller discrepancies, insoles are one of the most common intervention methods and have various advantages, such as non-invasiveness, low cost, easy application, and easy removal when unnecessary [9]. Insoles can also correct leg length discrepancy, relieve pain, and improve functional outcomes in patients with low back pain, hip osteoarthritis, or knee osteoarthritis [10].

Leg length discrepancy also causes asymmetry in the muscles related to the knee valgus. Asymmetry of leg length leads to deformation of the sacroiliac joint [11], and deformation of the sacroiliac joints causes tilting of the pelvis and lower back pain [12]. The knee joint is an intermediate joint of the lower extremity and is closely related to the alignment of the hip and ankle joints, which are adjacent [13]. The tilt and instability of the pelvis are associated with the weakness of the hip abductor muscles, and the gluteus medius is usually weakened in people with deformed hip joints or chronic back pain [14]. In other words, leg length discrepancy causes lateral pelvic tilt, which leads to abnormal gait, resulting in muscle imbalance, and the weaker leg side tilts the pelvis downward owing to the difference in the tension of the tensor fasciae latae. The tensor fasciae latae shows a pattern of activity that stabilizes the knee varus and knee valgus [15] most of the pelvic muscles are not activated to stabilize the knee against varus-valgus load, but the tensor fasciae latae exhibits substantial activity [15]. In a previous study, increased valgus motion and valgus moment in the knee joint during the impact phase of the jump landing operation were the major predictors of increased likelihood of the anterior cruciate ligament $(A C L)$ injury [16].

Correspondence address: Tae-Lim Yoon, Department of Physical Therapy, College of Health and Medical Science, Cheongju University, 298, Daeseong-ro, Cheongwon-gu, Cheongju-si, Chungcheongbuk-do, Republic of Korea, 28503, e-mail: taelimyoon@cju.ac.kr 
Subjects with large leg length discrepancies may experience an asymmetric effect in the alignment of the musculoskeletal system, such as the knee nevus angle, when weight is loaded. Previous studies evaluated the symmetry of weight load in subjects with leg length discrepancy and found that a discrepancy of more than $2 \mathrm{~cm}$ caused asymmetry of the ground repulsive force [7]. Clear gait asymmetry has been observed when there is a difference in leg length greater than $2.33 \mathrm{~cm}$ [17]. In other studies that created artificial leg length differences, there was no difference in ground repulsive force at a difference of $2.3 \mathrm{~cm}$ of leg length, but there was a difference in ground repulsive power at a difference of $3.5 \mathrm{~cm}$ [18]. In previous studies, a leg length discrepancy of $2 \mathrm{~cm}$ or more usually resulted in asymmetry.

However, there have been no studies investigating whether the application of insoles to high-intensity weight-bearing tasks, such as a drop jump task or a step landing task, has a significant effect on the knee valgus and related muscles in subjects with leg length discrepancies of less than $2 \mathrm{~cm}$. The purpose of this study was therefore to examine changes in the knee valgus angle, as well as the associated pelvic muscles, gluteus medius and tensor fasciae latae, when using an insole under high-intensity landing.

\section{Subjects and methods}

\section{Subjects}

Overall, 34 healthy people in their 20 s agreed to participate in the experiment. After 4 dropped out, 30 remaining subjects were selected. Of the 30 selected subjects, 3 were not able to participate owing to personal reasons. Thus, 27 subjects participated in the test; the criteria for selection were a leg length discrepancy of 5-20 mm [19], no pain or surgery in the joints of either lower limb, no history of orthopaedic or neurological disease or surgery, and no pain or clinically abnormal muscle strength or muscle tone resulting in abnormal walking. The general characteristics of the participants are presented in Table 1. Prior to the study, all subjects were fully briefed on the research methods.

Table 1. Characteristics of participants

\begin{tabular}{|l|c|}
\hline Variable & Group $(n=27)$ \\
\hline Age (years) & $21.11(1.42)$ \\
\hline Gender (F/M) & $13 / 14$ \\
\hline Height $(\mathrm{cm})$ & $167.28(7.17)$ \\
\hline Weight $(\mathrm{kg})$ & $61.23(8.87)$ \\
\hline Body mass index & $21.78(1.87)$ \\
\hline Leg length discrepancy $(\mathrm{cm})$ & $1.26(0.55)$ \\
\hline
\end{tabular}

\section{Measurements}

\section{Leg length discrepancy}

After aligning the centreline between the trunk, knee joints, and ankle joints in the supine position, the functional leg length was measured from the point at the lower middle of the navel to the medial malleolus of both legs by using a tape measure. The anatomical leg length was assessed from the anterior superior iliac spine to the medial malleolus of both legs. After measuring 3 times, the average value was recorded [20].

\section{Electromyography}

Electromyography (EMG) was performed by using surface EMG equipment (TeleMyo2400T, Noraxon Co., USA) with disposable electrodes (Electrode 2237, 3M, USA) made of silver/silver chloride (Ag/AgCl). To minimize errors in measurement, the hair was removed from the electrode attachment site to reduce skin resistance; impurities and foreign substances were removed with alcohol, and the electrode was attached after the alcohol had completely dried [21]. Two electrode contacts were applied to the gluteus medius, and the distance between the electrodes was $2 \mathrm{~cm}$. The gluteus medius electrode was placed $5 \mathrm{~cm}$ posterior to and $15 \mathrm{~cm}$ lower than the iliac apophysis [22]. For the tensor fasciae latae, 2 electrodes were attached between the iliotibial band and the middle half of the femur, $2 \mathrm{~cm}$ apart. All the electrodes were attached parallel to the direction of the muscle fibres and converted the surface EMG signals into digital signals with a multi-channel remote control system. The sampling rate of the EMG signals was set at $1000 \mathrm{~Hz}$. The bandwidth was measured by using a measurement frequency band of the surface EMG measuring equipment, $20-450 \mathrm{~Hz}$, and the sampled signal was processed with the root mean square method.

To standardize the muscle activity of the gluteus medius and tensor fasciae latae, the resistance was measured in the bare manual muscle test posture to induce maximum voluntary isometric contraction (MVIC), which was used to calculate \%MVIC. To prevent compensation during measurement, the participants held the bed with both hands in a supine position, and rest time was provided to reduce muscle fatigue after each motion. The MVIC value of each muscle was normalized by measuring the signal amount of the EMG for 5 seconds and averaging the middle 3 seconds of the measurement; this was repeated 3 times, and the average was used to calculate \%MVIC [23]. Peak EMG within each contraction (repetition) was normalized to the \%MVIC.

\section{mYOVIDEO}

In this study, the knee valgus angle was measured by using a video capture device (NiNOX ${ }^{\mathrm{TM}}$ 125, Noraxon Co., USA). The entire experiment was recorded by equipment installed at a height of $1 \mathrm{~m}$ at a point $4 \mathrm{~m}$ away from where the subjects landed during the tasks. The recorded video was set to 60 frames per second, with a resolution of $1088 \times 704$ pixels, and markers were attached to the centre of the femur, patella, and tibia of the subjects. From the marker attached to the patella, an imaginary line was drawn to each marker to measure the angle of the crossing point, and this was analysed by using the MR3.12 program (Noraxon Co., USA).

\section{Step landing task}

Before the experiment, a $30-\mathrm{cm}$ high bench was prepared and a point marked $30 \mathrm{~cm}$ away from the bench. Each participant stood with their feet on the front line of the bench. Then, they stepped forward, with the centre of the foot of the short leg landing at the marked point (Figure 1). The knee valgus angle was measured at the lowest knee height when landing [24]. The EMG peak at the moment when the subject's foot hit the floor was evaluated. 


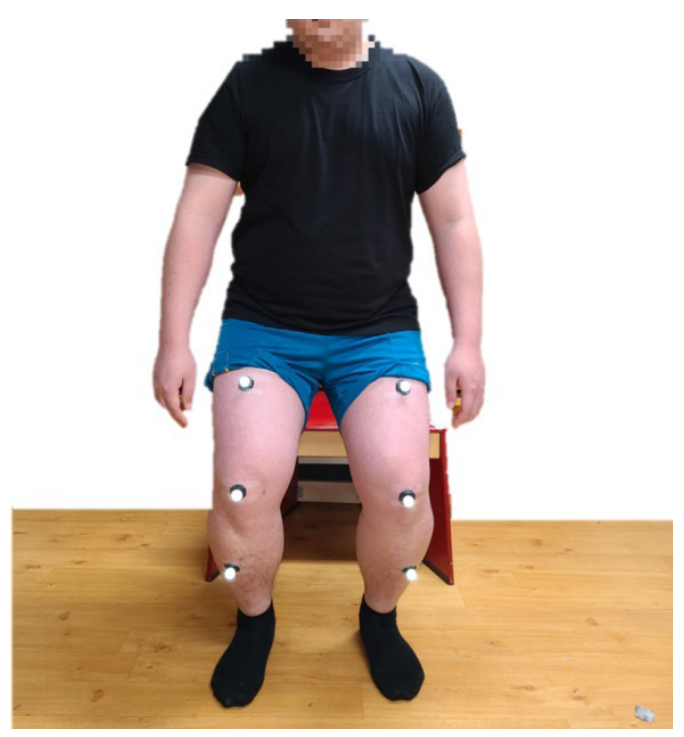

Figure 1. Example of step landing task

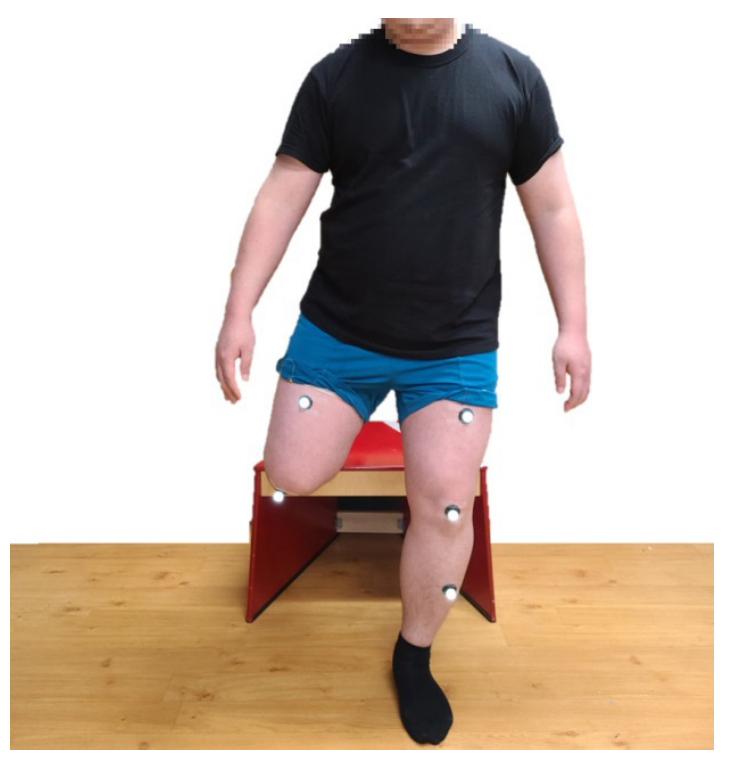

Figure 2. Example of drop jump task

\section{Drop jump task}

Before the experiment, a $30-\mathrm{cm}$ high bench was prepared and a point marked $30 \mathrm{~cm}$ away from the bench. Each participant stood with their feet on the front line of the bench. They then jumped and landed so that the marked point was between their feet. This was then repeated but swinging both arms to provide maximum recoil to jump vertically (Figure 2). The knee valgus angle was measured at the lowest knee height during the landing process after the second jump [24]. The EMG peak was evaluated after the second landing.

\section{Procedure}

The unilateral step landing task and bilateral drop jump task were used to model landing during daily life. After MVIC was measured as described above, the subjects listened to instructions for the 2 tasks and practised them 3 times to become acquainted with the procedure. Each patient was allowed to randomly select 1 from 2 folded pieces of paper located in a container and representing the 2 tasks. They per-

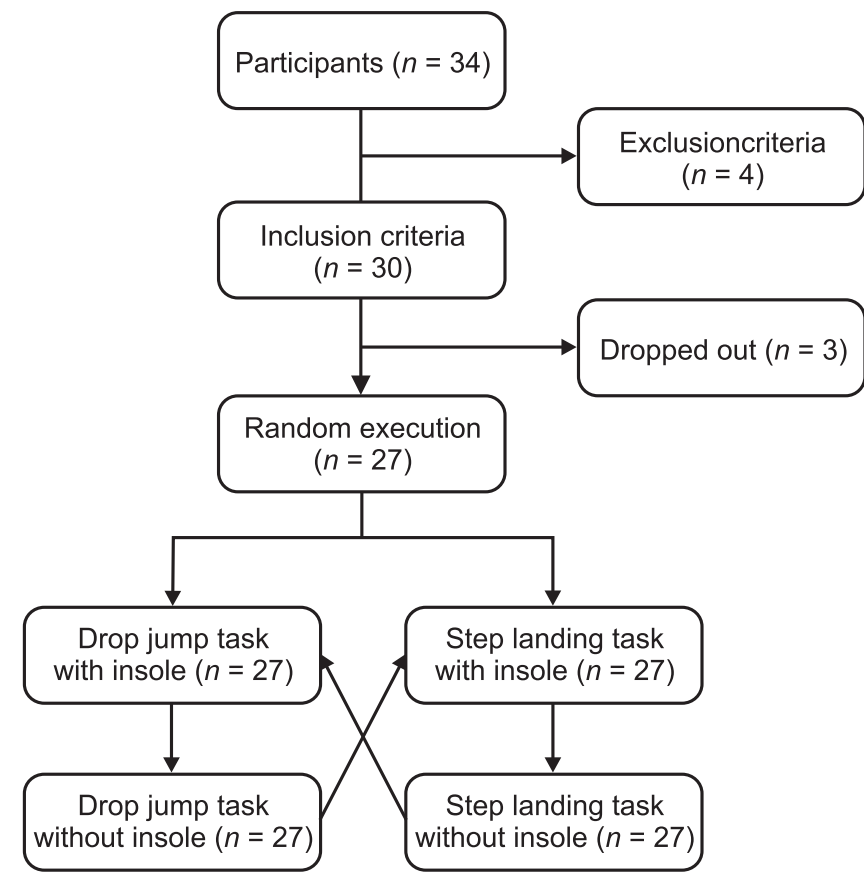

Figure 3. The study procedure flow chart

3 times without any insole (Figure 3). EMG and the knee valgus angles of the gluteus medius and tensor fasciae latae were recorded as the average values measured across the 3 repetitions. There were 5-minute intervals between the tests.

\section{Data analysis}

Sample size was determined by examining the pilot study. Power analysis (with $\mathrm{G}^{*}$ Power 3.0.10) revealed that 5 patients were sufficient to produce a power level of $95 \%$ with a detected effect size of 0.76 .

All measurements were analysed by using SPSS 22.0 for Windows. Paired $t$-tests were performed to compare the muscle activity of the gluteus medius and tensor fasciae latae and the knee valgus angle during the step landing and drop jump tasks with and without an insole. The significance level was set at $p<0.05$.

\section{Ethical approval}

The research related to human use has complied with all the relevant national regulations and institutional policies, has followed the tenets of the Declaration of Helsinki, and has been approved by the Cheongju University Institutional Review Board (approval number: 1041107-202004-HR-011-01).

\section{Informed consent}

Informed consent has been obtained from all individuals included in this study.

\section{Results}

There were no significant differences in the knee valgus angle between the insole and non-insole conditions during either the step landing task or the drop jump task (Table 2).

There were significant differences in the EMG peak between the insole and non-insole conditions in the tensor fasciae latae on the short leg side during the drop jump task and in the gluteus medius on the long leg side during the step landing task. There were no other significant differences (Table 3). 
Table 2. Results of the knee valgus angles with and without insole during both tasks

\begin{tabular}{|l|c|c|c|c|}
\hline \multirow{2}{*}{ Task } & \multicolumn{2}{|c|}{ Knee valgus angle $($ mean $\pm S D)$} & \multirow{2}{*}{$t$} & $p$ \\
\cline { 2 - 3 } & No insole & Insole & & \\
\hline Drop jump & $9.72(5.75)$ & $10.48(7.17)$ & -1.00 & 0.32 \\
\hline Step landing & $9.01(5.74)$ & $7.76(5.90)$ & 1.74 & 0.09 \\
\hline
\end{tabular}

Table 3. Results of the muscle activity with and without insole during both tasks

\begin{tabular}{|c|c|c|c|c|}
\hline \multirow{2}{*}{ Parameter } & \multicolumn{2}{|c|}{ Normalized EMG peak } & \multirow{2}{*}{$t$} & \multirow{2}{*}{$p$} \\
\hline & No insole & Insole & & \\
\hline Jump short leg Gmed & $84.66(30.86)$ & $82.98(36.52)$ & 0.27 & 0.78 \\
\hline Jump short leg TFL & $195.78(123.71)$ & $162.53(98.05)$ & 2.84 & $0.01^{*}$ \\
\hline Jump long leg Gmed & $94.62(72.62)$ & $85.80(48.00)$ & 1.19 & 0.24 \\
\hline Jump long leg TFL & 168.01 (107.52) & 156.19 (79.78) & 0.99 & 0.32 \\
\hline Step short leg Gmed & $28.91(19.59)$ & $26.60(18.74)$ & 0.89 & 0.38 \\
\hline Step short leg TFL & $50.76(31.51)$ & $49.32(31.98)$ & 0.34 & 0.73 \\
\hline Step long leg Gmed & $17.81(8.77)$ & $21.57(10.71)$ & -4.06 & $<0.01^{*}$ \\
\hline Step long leg TFL & $53.87(37.23)$ & $53.34(33.52)$ & 0.15 & 0.88 \\
\hline
\end{tabular}

EMG - electromyography, Gmed - gluteus medius, TFL - tensor fasciae latae

Values reported as mean peak normalized EMG \pm standard deviation in \% of the maximum voluntary isometric contraction

${ }^{*} p<0.05$

\section{Discussion}

In this study, we investigated changes in the knee valgus angle and the muscle activity of the gluteus medius and tensor fasciae latae during a drop jump task and a step landing task with and without insoles in people with leg length discrepancies. The tasks are the most common landing behaviours in everyday life. There were significant differences in the EMG peak between the insole and non-insole conditions in the tensor fasciae latae on the short leg side during the drop jump task and in the gluteus medius on the long leg side during the step landing task.

There was no significant difference in the knee valgus angle between the insole and non-insole conditions during either the step landing task or the drop jump task, which is in contrast to our hypothesis that we would find an increased knee valgus angle on the long leg side, leading to asymmetry on both sides. This unexpected result could be due to small leg length discrepancies in our study. In a previous study, gait analyses of groups with a leg length difference of about $1 \mathrm{~cm}$ and of less than $1 \mathrm{~cm}$ revealed symmetry and showed no significant differences [25]. However, another study indicated a significant increase in the knee joint angle on the long leg side in the stance phase during gait in a group with a difference in leg length of more than $2 \mathrm{~cm}$, but there was no significant difference in the knee joint angle of the short leg, regardless of the degree of leg length discrepancy [17]. In another previous study, the angle of the knee joint on the long leg side was significantly larger when the leg length discrepancy was $2 \mathrm{~cm}$ or more; this was because a leg length difference of less than $2 \mathrm{~cm}$ is mainly compensated for by the pelvis and hip joints [26]. Consistently among the previous studies, a leg length discrepancy of $2 \mathrm{~cm}$ or more had a compensation effect when landing through a change in the angle of the knee joint, but a difference in leg length of less than $2 \mathrm{~cm}$ showed no change in the knee joint angle, as sufficient compensation was seen in the pelvis and hip joints.
Most of the studies mentioned above used relatively light tasks under walking conditions. In our study, even if the leg length differences were small, it would be expected that a different result would be observed under the relatively heavy loads of jumping or landing tasks, but this was not the case. Therefore, there is no reason to consider the use of insoles for asymmetry of the knee valgus during jumping or landing among subjects whose leg length discrepancies are less than $2 \mathrm{~cm}$.

For the EMG peaks, there was significantly lower muscle activity in the tensor fasciae latae on the short leg side during the drop jump task with an insole than without it. In addition to affecting hip joint flexion and knee joint extension, the tensor fasciae latae is the only muscle that affects knee joint abduction and contributes to the creation of knee valgus moments [27]. In a similar study, the increase in the tensile load of the iliotibial band due to a strong contraction of the tensor fasciae latae was converted into compressive load outside the knee joint, and the knee valgus moment increased [28]. In another study, increasing tensor fasciae latae activity raised the knee valgus angle, and decreasing tensor fasciae latae activity reduced the knee varus moment [29]. Another similar study suggested that increasing the knee valgus moment raised the load on the medial collateral ligament and also increased the anterior tibial translation, which enlarged the probability of ACL injury [30]. Therefore, in our study, it can be inferred that the reduction in the activity of the tensor fasciae latae, which is the abductor muscle, results from a decrease in knee internal rotation or knee adduction moment owing to the insole. Overall, it is supposed that the significant decrease in tensor fasciae latae activity was partly due to the knee adduction moment reduction due to wearing the insole, but it did not make a significant difference in the knee valgus angle.

There was also significantly greater muscle activity in the gluteus medius on the long leg side during the step landing task with the insole than without it. As compared with the 
drop jump task, in which weight is supported with both legs, a larger knee valgus angle appears in the step landing task, in which weight is supported with only one [31]. Upon landing, the gluteus medius acts as a strong hip abductor, contributing to the stabilization of the pelvic tilt during the gait cycle, and the knee abduction moment increases as the activity of the gluteus medius increases [32]. It can be inferred that this is a result of a decrease in knee adduction moment due to wearing the insole. Despite these results, there was no significant difference in the knee valgus angle during the step landing task in our study.

In summary, there was no significant difference in the knee valgus angle during the high-intensity drop jump or step landing motions in a group of subjects with minor leg length discrepancies. However, it can be concluded that the significant decrease in tensor fasciae latae activity on the short leg side during the drop jump task and the significant increase in gluteus medius activity on the long leg side during the step landing task result from a decrease in knee adduction moment due to wearing the insole. Knee adduction moment during landing may cause non-contact ACL damage, and, even with a small leg length discrepancy, using an insole may help to prevent non-contact $A C L$ damage that can occur in high-intensity landing motions.

\section{Limitations}

There are 3 main limitations to this study. First, research involving participants in their 20 s is difficult to generalize to other age groups. Second, as the knee angle was viewed in 2 dimensions, there may have been a difference from the actual knee movement. Third, since a flat-type insole was used, there may be a difference when applying other types of insole. Future studies should seek to measure the knee angle more accurately by viewing it in 3 dimensions.

\section{Conclusions}

In this study, we investigated changes in the knee valgus angle and the activity of the gluteus medius and tensor fasciae latae during a drop jump task and a step landing task when an insole was used by subjects with leg length discrepancy. The results concerning knee valgus show that there is no reason to consider the use of an insole for knee valgus asymmetry during jump or landing in subjects with leg length discrepancies less than $2 \mathrm{~cm}$. However, the EMG peak implies that using an insole for individuals with leg length discrepancies can reduce the load on the medial collateral ligaments, which maintain knee stability by limiting the anterior tibial translation. This may lower the likelihood of frequent non-contact ACL damage on high-intensity landings.

\section{Acknowledgements}

This study was based on the master's thesis created by Seung-Eun Oh in 2021 at Cheongju University.

\section{Disclosure statement}

No author has any financial interest or received any financial benefit from this research.

\section{Conflict of interest}

The authors state no conflict of interest.

\section{References}

1. Blake RL, Ferguson H. Limb length discrepancies. J Am 7315-82-1-33.
2. Moseley CF. Leg length discrepancy. Orthop Clin North Am. 1987;18(4):529-535.

3. Schuit D, Adrian M, Pidcoe P. Effect of heel lifts on ground reaction force patterns in subjects with structural leglength discrepancies. Phys Ther. 1989;69(8):663-670; doi: 10.1093/ptj/69.8.663.

4. Papaioannou T, Stokes I, Kenwright J. Scoliosis associated with limb-length inequality. J Bone Joint Surg. 1982; 64(1):59-62; doi: 10.2106/00004623-198264010-00009.

5. Gurney B. Leg length discrepancy. Gait Posture. 2002; 15(2):195-206; doi: 10.1016/s0966-6362(01)00148-5.

6. Brady RJ, Dean JB, Skinner TM, Gross MT. Limb length inequality: clinical implications for assessment and intervention. J Orthop Sports Phys Ther. 2003;33(5):221234; doi: 10.2519/jospt.2003.33.5.221.

7. Kaufman KR, Miller LS, Sutherland DH. Gait asymmetry in patients with limb-length inequality. J Pediatr Orthop. 1996;16(2):144-150; doi: 10.1097/00004694-1996030 00-00002.

8. Stanitski DF. Limb-length inequality: assessment and treatment options. J Am Acad Orthop Surg. 1999;7(3): 143-153; doi: 10.5435/00124635-199905000-00001.

9. Campbell TM, Ghaedi BB, Tanjong Ghogomu E, Welch V. Shoe lifts for leg length discrepancy in adults with common painful musculoskeletal conditions: a systematic review of the literature. Arch Phys Med Rehabil. 2018; 99(5):981-993; doi: 10.1016/j.apmr.2017.10.027.

10. Golightly YM, Allen KD, Helmick CG, Renner JB, Jordan JM. Symptoms of the knee and hip in individuals with and without limb length inequality. Osteoarthritis Cartilage. 2009;17(5):596-600; doi: 10.1016/j.joca.2008.11. 005.

11. Cummings SR, Browner W, Black DM, Nevitt MC, Genant HK, Cauley J, et al. Bone density at various sites for prediction of hip fractures. The study of Osteoporotic Fractures Research Group. Lancet. 1993;341(8837):72-75; doi: 10.1016/0140-6736(93)92555-8.

12. Vink $P$, Kamphuisen $H A$. Leg length inequality, pelvic tilt and lumbar back muscle activity during standing. Clin Biomech. 1989;4(2):115-117; doi: 10.1016/0268-0033 (89)90049-1.

13. Chae J-B, Cho H-R, Hwa N-J, Kim Y-H. The change of gait as Q-angle in chronic knee osteoarthritis disease [in Korean]. J Korean Soc Phys Med. 2010;5(1):71-79.

14. Penney T, Ploughman M, Austin MW, Behm DG, Byrne $\mathrm{JM}$. Determining the activation of gluteus medius and the validity of the single leg stance test in chronic, nonspecific low back pain. Arch Phys Med Rehabil. 2014;95(10): 1969-1976; doi: 10.1016/j.apmr.2014.06.009.

15. Buchanan TS, Lloyd DG. Muscle activation at the human knee during isometric flexion-extension and varus-valgus loads. J Orthop Res. 1997;15(1):11-17; doi: 10.1002/ jor.1100150103.

16. Hewett TE, Myer GD, Ford KR, Heidt RS, Colosimo AJ, McLean SG, et al. Biomechanical measures of neuromuscular control and valgus loading of the knee predict anterior cruciate ligament injury risk in female athletes: a prospective study. Am J Sports Med. 2005;33(4):492501; doi: 10.1177/0363546504269591.

17. Liu XC, Fabry G, Molenaers G, Lammens J, Moens P. Kinematic and kinetic asymmetry in patients with leglength discrepancy. J Pediatr Orthop. 1998;18(2):187189; doi: 10.1097/01241398-199803000-00010.

18. Brand RA, Yack HJ. Effects of leg length discrepancies on the forces at the hip joint. Clin Orthop Relat Res. 1996; 333:172-180; doi: 10.1097/00003086-199612000-00017. 
19. Ashour R, Abdelraouf O, Abdallah A, Sweif R. Effect of footwear modification on postural symmetry and body balance in leg length discrepancy: a randomized controlled study. Int J Osteopath Med. 2019;32(3):13-20; doi: 10.1016/j.ijosm.2019.02.001.

20. Sabharwal S, Kumar A. Methods for assessing leg length discrepancy. Clin Orthop Relat Res. 2008;466(12):29102922; doi: 10.1007/s11999-008-0524-9.

21. Jeong J-G, Jang S-E, Kim Y-N, Hwang T-Y, Cho W-S, Kim S-H. The effect of flexibility, muscle strength and electrophysiologic nature on ballistic stretching [in Korean]. J Kor Acad Clin Elec. 2010;8(2):1-6; doi: 10.5627/ KACE.2010.8.2.001.

22. Nelson-Wong E, Gregory DE, Winter DA, Callaghan JP. Gluteus medius muscle activation patterns as a predictor of low back pain during standing. Clin Biomech. 2008; 23(5):545-553; doi: 10.1016/j.clinbiomech.2008.01.002.

23. Kim H-R, Song Y-J, Moon S-G, Jang H-J. The influence of electromyographic activation on gluteus medius and tensor fascia lata by functional leg length discrepancy in women's university students during lunge [in Korean]. J Korean Acad Orthop Man Phys Ther. 2013;19(2):39-46.

24. Herrington L, Munro A. Drop jump landing knee valgus angle; normative data in a physically active population. Phys Ther Sport. 2010;11(2):56-59; doi: 10.1016/j.ptsp. 2009.11.004.

25. Seeley MK, Umberger BR, Clasey JL, Shapiro R. The relation between mild leg-length inequality and able-bodied gait asymmetry. J Sports Sci Med. 2010;9(4):572-579.

26. Walsh $M$, Connolly $P$, Jenkinson $A$, O'Brien $T$. Leg length discrepancy - an experimental study of compensatory changes in three dimensions using gait analysis. Gait Posture. 2000;12(2):156-161; doi: 10.1016/s0966-6362 (00)00067-9.

27. Flaxman TE, Alkjær T, Simonsen EB, Krogsgaard MR, Benoit DL. Predicting the functional roles of knee joint muscles from internal joint moments. Med Sci Sports Exerc. 2017;49(3):527-537; doi: 10.1249/MSS.0000000 000001125.

28. Fairclough J, Hayashi K, Toumi H, Lyons K, Bydder G, Phillips N, et al. The functional anatomy of the iliotibial band during flexion and extension of the knee: implications for understanding iliotibial band syndrome. J Anat. 2006;208(3):309-316; doi: 10.1111/j.1469-7580.2006. $00531 . x$

29. Lloyd DG, Buchanan TS. Strategies of muscular support of varus and valgus isometric loads at the human knee. J Biomech. 2001;34(10):1257-1267; doi: 10.1016/ s0021-9290(01)00095-1.

30. Shelburne KB, Pandy MG, Torry MR. Comparison of shear forces and ligament loading in the healthy and ACL-deficient knee during gait. J Biomech. 2004;37(3): 313-319; doi: 10.1016/j.jbiomech.2003.07.001

31. Nejishima M, Urabe Y, Yokoyama S. Relationship between the knee valgus angle and EMG activity of the lower extremity in single- and double-leg landing. J Biomech. 2007;40(2):S743; doi: 10.1016/S0021-9290(07) 70731-5.

32. Kim D, Unger J, Lanovaz JL, Oates AR. The relationship of anticipatory gluteus medius activity to pelvic and knee stability in the transition to single-leg stance. PM R. 2016;8(2):138-144; doi: 10.1016/j.pmrj.2015.06.005. 